\title{
Eficácia de diferentes formulações de acaricidas sobre larvas de Rhipicephalus sanguineus (Latreille, 1806) (Acari: Ixodidae) e Rhipicephalus (Boophilus) microplus (Canestrini, 1887) (Acari: Ixodidae)
}

\author{
Luciano Melo de Souza ${ }^{1}$ \\ Marco Antonio de Andrade Belo ${ }^{1,2 *}$ \\ Ives Charlie da Silva ${ }^{2}$ \\ ${ }^{1}$ Universidade Camilo Castelo Branco, Campus de Descalvado, Descalvado - SP, Brasil \\ ${ }^{2}$ Departamento de Medicina Veterinária Preventiva \\ Universidade Estadual Paulista Júlio de Mesquita Filho \\ Via de acesso Prof. Paulo Donato Castellane, Km5, CEP 14.870-000, Jaboticabal - SP, Brasil \\ * Autor para correspondência \\ maabelo@hotmail.com
}

Submetido em 04/06/2016

Aceito para publicação em 23/11/2016

\section{Resumo}

O parasitismo por ácaros sempre foi objeto de preocupações e perdas relacionadas à produção animal, assim como à transmissibilidade de agentes etiológicos de importantes doenças; e aproximadamente $95 \%$ das formas evolutivas dos carrapatos em fase não parasitária encontram-se no ambiente. Com base na importância de se controlar estágios larvares dos ixodídeos no ambiente, este estudo avaliou a eficácia de acaricidas in vitro sobre larvas de Rhipicephalus (Boophilus) microplus e Rhipicephalus sanguineus. Foram testadas: Cipermetrina 15\% (T1); Deltametrina 25\% (T2); associação de Cipermetrina 5\% + Diclorvós 45\% + Butóxido de piperolina 25\% (T3); associação de Cipermetrina 15\% + Clorpiriphos 25\% + Citronela 1\% (T4) e Amitraz 12,5\% (T5). Para cada tratamento foram realizadas nove repetições contendo 20 larvas. As observações de motilidade foram realizadas: 1, 5, 10, 20 e 30 minutos após o tratamento (MPT); 1, 2, 6, 12 e $24 \mathrm{~h}$ após o tratamento (HPT); 5 dias após o tratamento (DPT). Os testes das larvas de $R$. (B.) microplus e $R$. sanguineus com deltametrina $25 \%$ serviram de controle positivo e demonstraram perda de motilidade de $100 \%$ das larvas com duas HPT para ambas espécies de ixodídeos. O R. (B.) microplus apresentou maior sensibilidade aos desafios contendo a cipermetrina $15 \%$ e $5 \%$ associada ao diclorvós e butóxido de piperonila. Larvas de $R$. sanguineus apresentaram maior sensibilidade em menor tempo ao tratamento com amitraz a 12,5\%. Contudo, apesar das diferenças no efeito acaricida observadas no decorrer dos tempos, obteve-se eficácia de 100\% para todos os compostos inseticidas sobre os instares das duas espécies $12 \mathrm{HPT}$, demonstrando que, quando utilizados nas doses recomendadas pelos fabricantes, os produtos comerciais podem ser eficazes em estratégias de controle ambiental das larvas.

Palavras chaves: Antiparasitários; Bovinos; Cães; Carrapatos; Ixodídeos; Larvicidas 


\section{Abstract}

Efficacy of acaricides against larvae of Rhipicephalus sanguineus (Latreille, 1806) (Acari: Ixodidae) and Rhipicephalus (Boophilus) microplus (Canestrini, 1887) (Acari: Ixodidae). Parasitism by mites has always been of concern with regard to losses in animal production and to the transmission of etiologic agents of important diseases as well, and about $95 \%$ of the developing forms of ticks in the non-parasitic phase are in the environment. In view of the importance of controlling larval stages of ticks in the environment, this study evaluated the efficacy of acaricides in vitro against larvae of Rhipicephalus (B.) microplus and $R$. sanguineus. Accordingly, we tested: $15 \%$ cypermethrin (T1); $25 \%$ deltamethrin (T2); combination of cypermethrin 5\% + $45 \%$ dichlorvos $+25 \%$ piperonyl butoxide (T3); combination of $15 \%$ cypermethrin $+25 \%$ chlorpyrifos $+1 \%$ citronella (T4); and 12.5\% amitraz (T5). In each treatment, there were nine replicates with 20 larvae each. The larvae were observed for motility at: 1, 5, 10, 20 and 30 minutes post-treatment (MPT); 1, 2, 6, 12 and 24 hours post-treatment (HPT); and 5 days post-treatment. Tests in $R$. (B.) microplus and $R$. sanguineus larvae with $25 \%$ deltamethrin served as a positive control and demonstrated $100 \%$ loss of motility at 2 HPT for both tick species. $R$. (B.) microplus larvae were most sensitive to treatments containing $15 \%$ cypermethrin or $5 \%$ cypermethrin combined with dichlorvos and piperonyl butoxide. Larvae of $R$. sanguineus were most sensitive to treatment with $12.5 \%$ amitraz. However, despite the differences in acaricidal effect during the observation time, $100 \%$ efficacy was obtained for all treatments against larvae of $R$. sanguineus and $R$. (B.) microplus at 12 HPT, demonstrating that appropriate doses of commercial acaricides can be effective in environmental control strategies against tick larvae.

Key words: Antiparasitic agents; Cattle; Dogs; Ixodids; Larvicide; Ticks

\section{Introdução}

O parasitismo por ácaros entremeando-se na história da humanidade e suas relações deletérias nas populações de animais domésticos sempre foi objeto de preocupações e perdas relacionadas à produção animal e à transmissibilidade de agentes etiológicos de importantes doenças zoonóticas (BELO et al., 2012; BRITES-NETO et al., 2015; SOUZA et al., 2015). Esses ácaros compreendem aproximadamente 800 espécies conhecidas, todos parasitos de vertebrados terrestres (DE LA FUENTE et al., 2010). Os prejuízos descritos na literatura científica, referente ao gênero Rhipicephalus (Boophilus) microplus, envolvem a perda de peso, diminuição da produção de leite, espoliação de sangue e transmissão de hemoparasitas, como Anaplasma spp., Babesia spp., sendo a espécie acarina monoxena considerada de ampla distribuição geográfica em países produtores de bovinos com danos econômicos que chegam a 3,24 bilhões de dólares no Brasil (GRISI et al., 2014). O Rhipicephalus sanguineus é um ácaro heteroxeno, cosmopolita, que parasita principalmente o cão doméstico e, secundariamente, outros mamíferos, aves e répteis (CAMARGO et al., 2016), sendo esse ácaro o principal vetor biológico das Rickettsias, destacando-se a Ehrlichia canis, agente etiológico da erliquiose canina.

Inúmeras estratégias são preconizadas para controlar os carrapatos $R$. sanguineus e $R$. microplus, entre elas o tratamento seletivo de hospedeiros e o controle estratégico (MOLENTO et al., 2013) focando principalmente no controle ambiental, pois cerca de $95 \%$ das formas evolutivas em fase não parasitária encontramse no ambiente (VERÍSSIMO, 2013). O emprego do controle biológico com fungos entomopatogênicos (CAMARGO et al., 2016), o uso de fitoterápicos (ADEHAN et al., 2016; ADENUBIA et al., 2016) e o uso de acaricidas comerciais para instalações de ordenha e canis (SOUZA et al., 2015) são descritos na literatura.

De maneira geral, o uso de produtos químicos aplicados diretamente nos animais continua sendo a principal forma de controle dos carrapatos e, segundo Souza et al. (2015), o uso indiscriminado de inseticidas em doses equivocadas tem se tornado um grave problema para a saúde dos animais, aumentando os resíduos ambientais e, no caso dos bovinos, colocando em risco a segurança alimentar por resíduos desses fármacos na carne e no leite, além de representar potencial fator de seleção e indução de resistência em cepas das duas espécies acarinas. 
Partindo-se da importância que representa o controle desses ixodídeos no ambiente, o presente estudo avaliou o efeito larvicida de formulações comerciais mais comumente utilizadas para controle do Rhipicephalus sanguineus e Riphiceplahus microplus.

\section{Material e Métodos}

\section{Material biológico}

Procedeu-se a coleta de fêmeas ingurgitadas de $R$. sanguineus e $R$. microplus em canis e em 10 propriedades leiteiras com infestações pelas respectivas espécies acarinas, no município de Descalvado (S2154'14" W47³7'12”), localizado no estado de São Paulo, Brasil.

Foram coletados apenas carrapatos de animais que não haviam recebido nenhum tipo de tratamento carrapaticida tópico por pelo menos 21 dias, injetável por pelo menos 45 dias e oral por pelo menos 60 dias antes da coleta, o que poderia interferir nos resultados.

As fêmeas ingurgitadas foram armazenadas em recipientes arejados e limpos, e posteriormente enviadas ao laboratório para cultivo e obtenção de larvas. No laboratório, tanto as fêmeas de $R$. sanguineus como as de $R$. (B.) microplus foram separadas de acordo com sua vitalidade e fixadas em placas de Petry para obtenção de ovos. Decorrido o período de postura, as massas produzidas foram recolhidas e armazenadas em seringas de $20 \mathrm{~mL}$ mantidas em estufa climatizada tipo B.O.D. sob temperatura de $27^{\circ} \mathrm{C}$ e $80 \pm 10 \%$ de umidade relativa para a obtenção dos instares larvares.

\section{Delineamento experimental}

Para a avaliação da perda de motilidade induzida pelos inseticidas, optou-se pelo método in vitro da pipeta de Pasteur, preconizado por Barci e Nogueira (2006) e adaptado por Souza et al. (2015). As larvas selecionadas para os bioensaios foram obtidas das oviposturas que apresentaram maior índice de eclodibilidade (>90\%). Previamente à exposição das larvas aos inseticidas, as pipetas foram impregnadas por imersão em solução contendo os inseticidas os quais foram diluídos em água destilada utilizando-se provetas volumétricas de $500 \mathrm{~mL}$, conforme diluições preconizadas pelos fabricantes e descritas na Tabela 1. Após a secagem natural, as larvas selecionadas de acordo com a motilidade foram aspiradas com bomba a vácuo para o interior das pipetas, sendo mantidas em estufa incubadora vertical (B.O.D). Em cada tratamento foram realizadas nove repetições contendo 20 larvas cada. As observações de motilidade foram realizadas: 1, 5, 10, 20 e 30 minutos após o tratamento (MPT); 1, 2, 6, 12 e 24 $\mathrm{h}$ após o tratamento (HPT). Após esse período, todas as pipetas foram colocadas na B.O.D entre 27 e $28^{\circ} \mathrm{C}$ de temperatura e umidade relativa (UR) de $80-90 \%$ para se completarem as observações após cinco dias.

TABELA 1: Compostos inseticidas empregados no estudo de inibição de motilidade de larvas de Rhipicephalus sanguineus e Rhipicephalus (Boophilus) microplus.

\begin{tabular}{|c|c|c|}
\hline Tratamentos $^{1}$ & Formulações ${ }^{2}$ & Diluições \\
\hline $\mathrm{T} 1$ & Cipermetrina $15 \%$ & $0,50 \mathrm{~mL} / 500 \mathrm{~mL}$ \\
\hline $\mathrm{T} 2$ & Deltametrina $25 \%$ & $0,50 \mathrm{~mL} / 500 \mathrm{~mL}$ \\
\hline $\mathrm{T} 3$ & Cipermetrina $5 \%+$ Diclorvós $45 \%+$ Butóxido de piperonila $25 \%$ & $1 \mathrm{~mL} / 500 \mathrm{~mL}$ \\
\hline $\mathrm{T} 4$ & Cipermetrina $15 \%+$ Clorpirifós $25 \%+$ Citronelal $1 \%$ & $1 \mathrm{~mL} / 500 \mathrm{~mL}$ \\
\hline T5 & Amitraz $12,5 \%$ & $0,5 \mathrm{~mL} / 500 \mathrm{~mL}$ \\
\hline T6 & Controle (água destilada) & $500 \mathrm{~mL}$ \\
\hline
\end{tabular}

${ }^{1}$ T1: Cypermil Pulverização ${ }^{\circledR}$ - Ouro Fino Saúde Animal, T2 - Butox ${ }^{\circledR}$ - MSD Saúde Anima, T3 - Cypermetril Plus ${ }^{\circledR}$ - Empresa Uzinas Chimicas Brasileiras S.A, T4 - Colosso ${ }^{\circledR}$ - Ouro Fino Saúde Animal, T5 - Triatox ${ }^{\circledR}$ - MSD Saúde Animal. ${ }^{2}$ Grupos químicos: Piretroides (Cipermetrina e Deltametrina); Organofosforados (Diclorvós e Clorpirifós); Formamidinas (Amitraz); Compostos orgânicos (Butóxido de piperonila) e Fitoterápicos (Citronelal). 


\section{Análise estatística}

Os resultados observados na mortalidade larvar foram analisados em delineamento inteiramente casualizado em parcelas subdividas no tempo, utilizando procedimento PROC GLM do programa SAS, versão 9.3 (SAS, 2012). A análise de variância para comparação das médias foi realizada pelo teste de $\mathrm{T}$ a $95 \%$ de confiança $(p<0,05)$ de acordo com Snedecor e Cochran (1980).

\section{Resultados}

As observações do efeito larvicida sobre os instares das duas espécies acarinas permitiram compilar dados individualizados nos tempos observacionais propostos para os bioensaios e podem ser visualizados para $R$. (B.) microplus e $R$. sanguineus nas Tabelas 2 e 3, respectivamente.
Os tratamentos com cipermetrina a $15 \%$ (T1) e com a associação desse piretroide ao diclorvós e ao butóxido de piperonila (BPO) (T3) resultaram em 100\% de perda da motilidade das larvas de $R$. microplus 60 minutos após o contato com os compostos (Tabela 2). Entretanto, 3,5\% das larvas do grupo da cipermetrina a $15 \%$ apresentaram motilidade transitória 6 horas pós-tratamento (HPT). Diferentemente, a deltametrina $25 \%$ (T2) e a associação de cipermetrina ao clorpirivós e citronela (T4) resultaram em inibição de $100 \%$ da motilidade após 2 horas, enquanto as larvas de $R$. (B.) microplus tratadas com amitraz (T5) apresentaram 100\% de paralisia somente depois de 12 horas de exposição ao inseticida (Tabela 2).

No estudo do efeito dos compostos farmacológicos sobre larvas do $R$. sanguineus (Tabela 3), o amitraz (T5) promoveu significativo efeito paralisante com

TABELA 2: Valores médios e análise de variância ${ }^{1}$ do número de larvas de Rhipicephalus (Boophilus) microplus imóveis expostas às diferentes formulações inseticidas.

\begin{tabular}{|c|c|c|c|c|c|c|c|c|}
\hline \multirow{2}{*}{$\begin{array}{c}\text { Período } \\
\text { experimental }\end{array}$} & \multicolumn{6}{|c|}{ Grupos experimentais / № de larvas mortas de Rhipicephalus (Boophilus) microphus ${ }^{2}$} & \multirow{2}{*}{$\begin{array}{l}\text { Valor } \\
\text { de } F^{3}\end{array}$} & \multirow{2}{*}{$\operatorname{Pr}>F^{3}$} \\
\hline & T1 & $\mathbf{T 2}$ & T3 & T4 & T5 & T6 & & \\
\hline 1 minuto & $0,0 \mathrm{Ae}$ & $0,0 \mathrm{Ac}$ & $0,0 \mathrm{Ac}$ & $0,0 \mathrm{Ac}$ & $0,0 \mathrm{Ac}$ & $0,0 \mathrm{Aa}$ & 0,00 & 1,000 \\
\hline 5 minutos & $0,0 \mathrm{Ae}$ & $0,0 \mathrm{Ac}$ & $0,0 \mathrm{Ac}$ & $0,0 \mathrm{Ac}$ & $0,0 \mathrm{Ac}$ & $0,0 \mathrm{Aa}$ & 0,00 & 1,000 \\
\hline 10 minutos & $3,3 \mathrm{Ad}$ & $0,0 \mathrm{Ac}$ & $0,0 \mathrm{Ac}$ & $0,0 \mathrm{Ac}$ & $0,0 \mathrm{Ac}$ & $0,0 \mathrm{Aa}$ & 1,95 & 0,0908 \\
\hline 20 minutos & $6,7 \mathrm{Bc}$ & $0,0 \mathrm{Cc}$ & $14,3 \mathrm{Ab}$ & $0,0 \mathrm{Cc}$ & $0,0 \mathrm{Cc}$ & $0,0 \mathrm{Ca}$ & 37,09 & $<0,0001$ \\
\hline 30 minutos & $12,0 \mathrm{Bb}$ & $4,7 \mathrm{Cb}$ & $16,3 \mathrm{Ab}$ & $17,3 \mathrm{Ab}$ & $0,0 \mathrm{Dc}$ & $0,0 \mathrm{Da}$ & 65,34 & $<0,0001$ \\
\hline 1 hora & $20,0 \mathrm{Aa}$ & $20,0 \mathrm{Aa}$ & $20,0 \mathrm{Aa}$ & $20,0 \mathrm{Aa}$ & 7,3 Bb & $0,0 \mathrm{Ca}$ & 74,78 & $<0,0001$ \\
\hline 2 horas & $20,0 \mathrm{Aa}$ & 19,3 Aa & $20,0 \mathrm{Aa}$ & $20,0 \mathrm{Aa}$ & $13,0 \mathrm{Ba}$ & $0,0 \mathrm{Ca}$ & 68,86 & $<0,0001$ \\
\hline 6 horas & $19,3 \mathrm{Aa}$ & $20,0 \mathrm{Aa}$ & $20,0 \mathrm{Aa}$ & $20,0 \mathrm{Aa}$ & $17,0 \mathrm{Aa}$ & $0,0 \mathrm{Ba}$ & 66,46 & $<0,0001$ \\
\hline 12 horas & $20,0 \mathrm{Aa}$ & $20,0 \mathrm{Aa}$ & $20,0 \mathrm{Aa}$ & $20,0 \mathrm{Aa}$ & $20,0 \mathrm{Aa}$ & $0,0 \mathrm{Ba}$ & 70,09 & $<0,0001$ \\
\hline 24 horas & $20,0 \mathrm{Aa}$ & $20,0 \mathrm{Aa}$ & $20,0 \mathrm{Aa}$ & $20,0 \mathrm{Aa}$ & $20,0 \mathrm{Aa}$ & $0,0 \mathrm{Ba}$ & 70,09 & $<0,0001$ \\
\hline 5 dias & $20,0 \mathrm{Aa}$ & $20,0 \mathrm{Aa}$ & $20,0 \mathrm{Aa}$ & $20,0 \mathrm{Aa}$ & $20,0 \mathrm{Aa}$ & $0,0 \mathrm{Ba}$ & 70,09 & $<0,0001$ \\
\hline Valor de $F^{3}$ & 79,63 & 105,47 & 85,00 & 100,11 & 89,42 & 0,00 & & \\
\hline $\operatorname{Pr}>F^{3}$ & $<0,0001$ & $<0,0001$ & $<0,0001$ & $<0,0001$ & $<0,0001$ & 1,000 & & \\
\hline
\end{tabular}

${ }^{1}$ Médias $(\mathrm{n}=9$ repetições de 20 larvas cada) seguidas pela mesma letra não diferem entre si pelo teste $\mathrm{T}(\mathrm{P}<0,05)$, letras maiúsculas na linha para comparação entre os tratamentos nos diferentes dias experimentais, e minúsculas nas colunas para avaliação da evolução de cada tratamento ao longo do tempo. ${ }^{2} \mathrm{~T} 1=$ Cipermetrina a $15 \%$; T2 = Deltametrina a $25 \%$; T3 = Cipermetrina 5\% + Diclorvós 45\% + Butóxido de piperolina 25\%; T4 = Cipermetrina 15\% + Clorpirivos 25\% + Citronela 1\%; T5 = Amitraz 12,5\%; T6 = Controle (água destilada). ${ }^{3}$ Valor do Teste F; Pr $>\mathrm{F}-$ Probabilidade de significância do valor de F. 
TABELA 3: Valores médios e análise de variância ${ }^{1}$ do número de larvas de Rhipicephalus sanguineus imóveis expostas às diferentes formulações inseticidas.

\begin{tabular}{|c|c|c|c|c|c|c|c|c|}
\hline \multirow{2}{*}{$\begin{array}{c}\text { Período } \\
\text { experimental }\end{array}$} & \multicolumn{6}{|c|}{ Grupos experimentais / № de larvas de Rhipicephalus sanguineus ${ }^{2}$} & \multirow{2}{*}{ Valor de $\mathbf{F}^{3}$} & \multirow{2}{*}{$\operatorname{Pr}>F^{3}$} \\
\hline & T1 & $\mathbf{T 2}$ & T3 & T4 & T5 & T6 & & \\
\hline 1 minuto & $0 \mathrm{Bd}$ & $0 \mathrm{Bd}$ & $0 \mathrm{Bf}$ & $0 \mathrm{Bc}$ & $20 \mathrm{Aa}$ & $0 \mathrm{Ba}$ & 297,74 & $<0,0001$ \\
\hline 5 minutos & $2 \mathrm{Cd}$ & $8 \mathrm{Bc}$ & $2 \mathrm{Ce}$ & $0 \mathrm{Dc}$ & $20 \mathrm{Aa}$ & $0 \mathrm{Da}$ & 269,16 & $<0,0001$ \\
\hline 10 minutos & $6 \mathrm{Cc}$ & $8 \mathrm{Bc}$ & $4 \mathrm{Dd}$ & $0 \mathrm{Ec}$ & $20 \mathrm{Aa}$ & $0 \mathrm{Ea}$ & 245,94 & $<0,0001$ \\
\hline 20 minutos & $6 \mathrm{Cc}$ & $10 \mathrm{Bb}$ & $6 \mathrm{Cc}$ & $0 \mathrm{Dc}$ & $20 \mathrm{Aa}$ & $0 \mathrm{Da}$ & 248,32 & $<0,0001$ \\
\hline 30 minutos & $8 \mathrm{Cc}$ & $10 \mathrm{Bb}$ & $8 \mathrm{Cb}$ & $8 \mathrm{Cb}$ & $20 \mathrm{Aa}$ & $0 \mathrm{Da}$ & 184,01 & $<0,0001$ \\
\hline 1 hora & $16 \mathrm{Bd}$ & $11,7 \mathrm{Cb}$ & $18 \mathrm{Aa}$ & $8 \mathrm{Db}$ & $20 \mathrm{Aa}$ & $0 \mathrm{Ea}$ & 246,22 & $<0,0001$ \\
\hline 2 horas & $20 \mathrm{Aa}$ & $20 \mathrm{Aa}$ & $20 \mathrm{Aa}$ & $20 \mathrm{Aa}$ & $6 \mathrm{Bc}$ & $0 \mathrm{Ca}$ & 360,81 & $<0,0001$ \\
\hline 6 horas & $20 \mathrm{Aa}$ & $20 \mathrm{Aa}$ & $20 \mathrm{Aa}$ & $20 \mathrm{Aa}$ & $14 \mathrm{Bb}$ & $0 \mathrm{Ca}$ & 288,81 & $<0,0001$ \\
\hline 12 horas & $20 \mathrm{Aa}$ & $20 \mathrm{Aa}$ & $20 \mathrm{Aa}$ & $20 \mathrm{Aa}$ & $20 \mathrm{Aa}$ & $0 \mathrm{Ba}$ & 297,74 & $<0,0001$ \\
\hline 24 horas & $20 \mathrm{Aa}$ & $20 \mathrm{Aa}$ & $20 \mathrm{Aa}$ & $20 \mathrm{Aa}$ & $20 \mathrm{Aa}$ & $0 \mathrm{Ba}$ & 297,74 & $<0,0001$ \\
\hline 5 dias & $20 \mathrm{Aa}$ & $20 \mathrm{Aa}$ & $20 \mathrm{Aa}$ & $20 \mathrm{Aa}$ & $20 \mathrm{Aa}$ & $0 \mathrm{Ba}$ & 297,74 & $<0,0001$ \\
\hline Valor de $\mathbf{F}^{3}$ & 296,88 & 215,18 & 318,32 & 404,07 & 87,37 & 0,000 & & \\
\hline $\operatorname{Pr}>F^{3}$ & $<0,0001$ & $<0,0001$ & $<0,0001$ & $<0,0001$ & $<0,0001$ & 1,000 & & \\
\hline
\end{tabular}

${ }^{1}$ Médias ( $\mathrm{n}=9$ repetições de 20 larvas cada) seguidas pela mesma letra não diferem entre si pelo teste $\mathrm{T}(\mathrm{P}<0,05)$, letras maiúsculas na linha para comparação entre os tratamentos nos diferentes dias experimentais, e minúsculas nas colunas para avaliação da evolução de cada tratamento ao longo do tempo. ${ }^{2} \mathrm{~T} 1$ = Cipermetrina a $15 \%$; T2 = Deltametrina a $25 \%$; T3 = Cipermetrina 5\% + Diclorvós $45 \%$ + Butóxido de piperolina 25\%; T4 = Cipermetrina 15\% + Clorpirivos 25\% + Citronela 1\%; T5 = Amitraz 12,5\%; T6 = Controle (água destilada). ${ }^{3}$ Valor do Teste F; $\operatorname{Pr}>\mathrm{F}-$ Probabilidade de significância do valor de F.

$100 \%$ de perda de motilidade em um minuto póstratamento, porém $70 \%$ das larvas apresentaram recuperação transitória da motilidade $2 \mathrm{HPT}$. O efeito do amitraz sobre a perda de motilidade das larvas foi progressivamente aumentando, alcançando $70 \% 6 \mathrm{HPT}$ e $100 \% 12$ HPT.

Os testes com cipermetrina $15 \%$ (T1), deltametrina $25 \%$ (T5) e com duas associações de piretroides e organofosforados (T3 e T4) demonstraram perda de motilidade em $100 \%$ das larvas de $R$. sanguineus 2 HPT (Tabela 3). O estudo comparativo da resposta de motilidade das larvas de $R$. (B.) microplus e $R$. sanguineus (Tabelas 2 e 3 ) demonstrou perda da mortalidade de $100 \%$ das larvas a partir de 12 HPT para todas as formulações testadas.

\section{Discussão}

A técnica utilizada na presente investigação, método in vitro utilizando-se a pipeta de Pasteur, preconizado por Barci e Nogueira (2006) e modificado por Souza et al. (2015), possibilitou a observação do efeito inseticida ao longo do tempo, pois permitiu facilmente o exame da perda de motilidade larval, pelo fato de a pipeta ser transparente, diferentemente da técnica de pacotes de larvas (Larval Packet Test) preconizada pela FAO (2004), na qual deve ser realizada a abertura de cada envelope para avaliação da motilidade das larvas nos diferentes períodos observados.

Desde o desenvolvimento do primeiro piretroide sintético na década de 1970, esses compostos vêm sendo largamente utilizados na agricultura mundial. 
A deltametrina apresenta apenas a substituição do átomo de cloro da bioresmetrina pelo bromo, proporcionando a fotoestabilidade a esse fármaco, que atualmente é o mais utilizado para o controle de carrapatos no ambiente, sendo a droga de eleição para o manejo sanitário de canis e instalações rurais. Neste contexto, os testes das larvas de $R$. (B.) microplus e $R$. sanguineus com esse piretroide serviram de controle positivo para análise comparativa dos resultados de motilidade, pois, para ambas as espécies de ácaros, a perda de motilidade de 100\% das larvas ocorreu 120 minutos depois do tratamento com deltametrina a $25 \%$. Resultados similares foram constatados por Fernandes et al. (2001), que estudaram os efeitos toxicológicos da deltametrina em $R$. sanguineus e encontraram incoordenação e paralisia seguida por morte. Mendes et al. (2007) observaram eficácia superior da deltametrina quando comparada com cipermetrina em estudos de imersão de adultos. Valores diferentes foram observados por Borges et al. (2007), em que a maioria das cepas de $R$. sanguineus testadas em Goiânia apresentaram resistência à cipermetrina, à deltametrina e ao coumafós.

Dessa forma, foi possível observar maior perda de motilidade nas larvas de $R$. (B.) microplus submetidas ao tratamento com Cipermetrina + Diclorvós + Butóxido de piperonila, alcançando eficácia de 100\% em 60 minutos após o tratamento. O Diclorvós pertencente ao grupo químico dos organofosforados e é muito utilizado há décadas no controle de pragas na agricultura, conhecido por inibir irreversivelmente a enzima acetilcolinesterase (AChE); já os piretroides, introduzidos no mercado brasileiro nos anos 80 , possuem propriedades lipofílicas que facilitam a penetração na cutícula dos carrapatos, após absorção são carreados pela hemolinfa até as células nervosas e seu sítio de ação é nos canais de sódio que são modulados pela proteína $\mathrm{C}$-quinase, responsável pelo controle da excitabilidade. Além disso, há evidências de que a deltametrina pode atuar como agonista colinérgico (GABA).

Com o aumento dos relatos de resistência acarina, a indústria farmacêutica veterinária vem buscando associar diferentes princípios ativos para potencializar a eficácia acaricida das formulações, entre essas estratégias de associações estão as formulações contendo organofosforados e piretroides. No presente estudo, para larvas do $R$. sanguineus, testadas com associação de Cipermetrina + Clorpiriphos + Citronela, não se observou efeito acaricida sinérgico, corroborando os achados de Soares et al. (2009), que também não observaram aumento de eficácia terapêutica dessa associação no controle de $R$. (B.) microplus em bovinos naturalmente infestados. Porém, esses autores descreveram o efeito potencializado das formulações de organofosforados e piretroides contendo o BPO. De acordo com Belo et al. (2012), o BPO atua na principal via de desintoxicação dos carrapatos, provocando a degradação oxidativa no sistema do citocromo P-450, potencializando os efeitos dos piretroides, inibindo os mecanismos naturais de defesa dos ácaros, resultando em efeito sinérgico e aumento da eficácia terapêutica das associações.

O estudo comparativo do efeito do amitraz sobre larvas das duas espécies acarinas revelou resultados bem distintos, demonstrando maior sensibilidade do carrapato dos canídeos a esse ativo. A farmacodinâmica das formamidinas em mamíferos demonstrou ações sobre receptores alfa-adrenérgicos, interação com receptores de octopamina no sistema nervoso central, inibição da monoaminaoxidases (MAO) e alterações na síntese de prostaglandinas (PINO et al., 2015). Borges et al. (2007) pesquisaram índices de resistência para o amitraz no estado de Goiás, usando o teste de imersão de larvas, e verificaram a sensibilidade de cepas $R$. sanguineus a essa formamidina. Louly et al. (2004) também observaram o efeito superior do amitraz sobre o $R$. sanguineus, corroborando os achados da presente pesquisa.

Entretanto, tal efeito não foi notado sobre as larvas de $R$. (B.) microplus do presente estudo, e a menor sensibilidade às formamidinas pode ser explicada pelo uso indiscriminado no Brasil desse fármaco para o controle de carrapatos nos bovinos, fato que pode justificar um maior nível de resistência das larvas. Santos et al. (2009) avaliaram o uso de amitraz em cepas de $R$. (B.) microplus obtidas em 100 propriedades no Rio Grande do Sul e verificaram baixa suscetibilidade desse fármaco no controle dos carrapatos dos bovinos.

Chama-se a atenção para o fenômeno momentâneo de ação do amitraz sobre lavas de $R$. sanguineus entre duas e seis horas após o tratamento para o amitraz $12,5 \%$ 
(T5), que, após ter exibido efeito de paralisia em 100\% das larvas do primeiro minuto observacional até os 60 ', um pequeno grupo delas recuperou a motilidade transitoriamente. Souza et al. (2015) observaram o efeito tóxico semelhante sobre a motilidade das larvas de Amblyomma cajennese submetidas ao tratamento com amitraz $12,5 \%$, ocorrendo recuperação transitória da capacidade motora. Outro estudo demostrou o efeito transitório para esse princípio ativo quando avaliavam a atividade in vitro do amitraz, fipronil e permetrina sobre larvas de Rhipicephalus bursa (CASTELLÀ-SPUNY; SOUZA, 1998).

As larvas em pipetas impregnadas com água destilada demonstraram $100 \%$ de motilidade ao longo do estudo. Barci e Nogueira (2006), utilizando a mesma técnica, observaram alterações significativas nos percentuais de mortalidade em larvas dos gruposcontrole apenas após o $20^{\circ}$ dia de avaliação.

Vale ressaltar que, apesar das diferenças nos efeitos larvicidas observadas no decorrer dos tempos observacionais, obteve-se eficácia de $100 \%$ para todas as formulações inseticidas contra larvas de $R$. sanguineus $\mathrm{e}$ $R$. (B.) microplus $12 \mathrm{~h}$ após a exposição, demonstrando que quando utilizados em doses indicadas pelos fabricantes os produtos comerciais são eficazes para o controle ambiental das larvas e poderiam ser utilizados no manejo sanitário de galpões, canis, instalações de ordenha, entre outros.

\section{Referências}

ADEHAN, S. B.; BIGUEZOTON, H.; ADAKAL, F.; DOSSA, T. J.; DOUGNON, E.; YOUSSAO, P.; SESSOU, A. B.; ABOH, A. K. I.; YOUSSAO, N.; ASSOGBA, G. A. Acaricidal activity of ethanolic and volatile extracts of the leaves of selected plants used in veterinary pharmacopeia on the larvae of Rhipicephalus microplus in Benin. Alexandria Journal of Veterinary Sciences, Alexandria, v. 49, n. 1, p. 1-11, 2016.

ADENUBIA, O. T.; FASINAB, F. O.; MCGAWA, L. J.; ELOFFA, J. N.; NAIDOO, C. V. Plant extracts to control ticks of veterinary and medical importance: a review. South African Journal of Botany, Pretoria, v. 105, n. 19, p. 178-193, 2016.

BARCI, L. A. G.; NOGUEIRA, A. H. C. Método para avaliação de mortalidade de larvas de Boophilus microplus (Canestrini, 1887) submetidas a tratamentos com produtos carrapaticidas. Arquivos do Instituto Biológico, São Paulo, v. 73, n. 1, p. 105-109, 2006.

BELO, M. A. A.; PRADO, E. J. R.; SOARES, V. E.; SOUZA, L. M.; MOTA, F. C. C.; GIAMLORENÇO, T. F.; GÍRIO, T. M. S. Eficácia de diferentes formulações no controle da mosca Haematobia irritans em bovinos naturalmente infestados. Bioscience Journal, Uberlândia, v. 28, p. 245-250, 2012.

BORGES, L. M. F.; SOARES, S. F.; FONSECA, I. N.; CHAVES, V. V.; LOULY, C. C. B. Acaricide resistance in Rhipicephalus sanguineus (Acari: Ixodidae) larvae from Goiania - GO, Brazil. Revista de Patologia Tropical, Goiânia, v. 36, n. 1, p. 1-1, 2007.

BRITES-NETO, J.; DUARTE, K. M. R., MARTINS, T. F. Tickborne infections in human and animal population worldwide, Veterinary World, Wankaner, v. 8, n. 3, p. 301-315, 2015.

CAMARGO, M. G.; NOGUEIRA, M. R. S.; MARCIANO, A. F.; COUTINHO-RODRIGUES, C. J. B.; SCOTT, F. B; ANGELO, I. C; PRATA, M. C. A.; BITTENCOURT, V. R. E. P. Metarhizium anisopliae for controlling Rhipicephalus microplus ticks under field conditions. Veterinary Parasitology, Amsterdam, v. 223, n.15, p. 38-42, 2016.

CASTELLÀ-ESPUNY, J.; SOUZA, L. M. Actividade in vitro del amitraz, friponil y permetrina, frente a larvas de Rhipicephalus bursa. In: SIMPÓSIO IBÉRICO SOBRE IXOIDIDEA ENFERMEDADES TRANSMISIBILES, VI, 1998, Setubal. Anais... Setubal: SISIET, 1998. Versão eletrônica.

DE LA FUENTE, J.; MANZANO-ROMAN, R.; NARANJO, V.; KOCAN, K. M.; IVKOVIC, Z.; BLOUIN, E. F. Identification of protective antigens by RNA interference for control of the lone star tick, Amblyomma americanum. Vaccine, Kidlinton, v. 28, n. 7, p. 1786-1795, 2010.

FAO. Resistance management and integrated parasite control in ruminants - Guidelines, Module 1 - Ticks: acaricide resistance: diagnosis, management and prevention. Rome: Food and Agriculture Organization, Animal Production and Health Division, 2004. $25 \mathrm{p}$.

FERNANDES, F. F.; FREITAS, E. P.; SILVA, J. R.; SILVA, O. R.; SILVA, I. G. Toxic effects and in vitro inefficacy of deltamethrin on larvae of Rhipicephalus sanguineus from Goiania, Goias, Brazil. Revista de Patologia Tropical, Goiânia, v. 34, p. 159-165, 2001.

GRISI, L.; LEITE, R. C.; MARTINS, J. R. S. Reassessment of the potential economic impact of cattle parasites in Brazil. Revista Brasileira de Parasitologia Veterinária, São Carlos, v. 23, n. 2, p. 150-156, 2014.

LOULY, C. C. B.; FONSECA, I. N.; OLIVEIRA, V. F.; MENEZES, L. B.; BORGES, L. M. F. Eficácia da flumetrina no controle de Rhipicephalus sanguineus em cães do canil da polícia militar do município de Goiânia-Goiás. Revista Brasileira de Parasitologia Veterinária, São carlos, v. 13, supl. 1, p. 321, 2004.

MENDES, M. C.; LIMA, C. K. P.; PRADO, A. P. Determinação da freqüência de realização de bioensaios para o monitoramento da resistência do carrapato Boophilus microplus (Acari: Ixodidae). Arquivos do Instituto Biológico, São Paulo, v. 74, n. 2, p. 87-93, 2007.

MOLENTO, M. B; FORTES, F. S; BUZATTI, A.; KLOSTER, F. S.; SPRENGER, L. K.; COIMBRA, E.; SOARES, L. D. Partial selective treatment of Rhipicephalus microplus and breed resistance variation in beef cows in Rio Grande do Sul, Revista Brasileira de Parasitologia Veterinária, São Carlos, v. 192, n. 1-3, p. 234-239, 2013.

PINO, J. D.; MOYANO-CIRES, P. V.; ANADON, M. J.; DÍAZ, M. J.; LOBO, M.; CAPO, M. A.; FREJO, M. T. Molecular mechanisms 
of amitraz mammalian toxicity: a comprehensive review of existing data. Chemical Research in Toxicology, Washington, v. 28, n. 6, p. 1073-1094, 2015.

SANTOS, T. G. B.; FARIAS, N. A. R.; CUNHA-FILHO, N. A.; PAPPEN, F. G.; VAZ-JUNIOR, I. S. Abordagem sobre o controle do carrapato Rhipicephalus (Boophilus) microplus no sul do Rio Grande do Sul. Pesquisa Veterinária Brasileira, Seropédica, v. 29, n. 1, p. 65-70, 2009.

SAS. SAS/STAT software changes and enhancements though [computer program]. Release 9.3. Cary: SAS Institute, 2012.

SOARES, V. E.; BELO, M. A. A.; SOUZA, L. M.; GUIARO, C. R.; BORTOLETTO NETO, O.; GIRIO, T. M. S.. Associação de cipermetrina, diclorvós e butóxido de pieronila contra Rhipicephalus (Boophilus) microplus em bovinos naturalmente infestados. Archives of Veterinary Science, Curitiba, v. 14, p. 1-8, 2009.
SOUZA, L. M; AMBROZIO, R. M.; SILVA, I. C.; BELO, M. A. A; CHIQUITO, D. Eficácia da deltrametrina no controle de larvas de Amblyomma cajennense (Fabrícius, 1787) (Acari: Ixodidae). Enciclopédia Biosfera, Goiânia, v. 11, p. 1120, 2015.

SNEDECOR, G. W.; COCHRAN, W. G. Statistical methods. Iowa: Iowa State University Press, 1980. 75 p.

VERÍSSIMO, C. J. Controle de carrapatos nas pastagens. Nova Odessa: Instituto de Zootecnia, 2013. 99 p. 